\title{
Color ptychography with a hyperspectral X-ray camera
}

\author{
Wiebe Stolp ${ }^{\mathrm{a}}$, Silvia Cipiccia ${ }^{\mathrm{b}, \mathrm{c}}$, Sander Vanheule ${ }^{\mathrm{a}}$, Darren Batey ${ }^{\mathrm{b}}$, Frederic van Assche ${ }^{\mathrm{a}}$, and \\ Matthieu N. Boone ${ }^{\text {a }}$ \\ ${ }^{a}$ UGCT-RP, Dept. Physics and Astronomy, Ghent University, Belgium. \\ ${ }^{\mathrm{b}}$ Diamond Light Source, Harwell Science and Innovation Campus, Fermi Avenue, Didcot, UK. \\ ${ }^{\mathrm{c}}$ Department of Medical Physics and Biomedical Engineering, University College London, \\ London, UK.
}

\begin{abstract}
A possible improvement on a new method of single acquisition hyperspectral (spectroscopic) ptychographic imaging, making use of a hyperspectral X-ray camera, is presented. Synchrotron undulator tapering is used to broaden the energy distribution of the X-ray beam to a suitable level. The combination of a coherent imaging method such as ptychography with spectroscopy poses difficulties in experimental setup design regarding probe size. The final goal of the experiment, a K-edge subtraction, is not achieved, but the technique is nevertheless promising. We discuss the problems and their possible solutions.
\end{abstract}

Keywords: spectroscopy, ptychography, coherent diffraction imaging, synchrotron, edge subtraction

\section{INTRODUCTION}

\subsection{Ptychography}

Ptychography is a diffractive imaging method that uses many overlapping adjacent coherently illuminated sample areas to iteratively reconstruct a complex image of both the object and the illuminating probe. The modulus of the object corresponds to its attenuating properties and the phase to the electron density of the sample. Ptychography and conventional Coherent Diffraction Imaging (CDI) are theoretically diffraction limited. Compared to conventional CDI, it allows imaging of extended samples. Similar to CDI, it requires a coherent illumination that is usually only available at synchrotron facilities.

\subsection{Hyperspectral ptychography}

Multi-spectral ptychography has been demonstrated before by scanning a sample area for each desired wavelength separately and combining the different reconstructions to feature an energy dimension. ${ }^{1}$ Our group has furthermore successfully demonstrated a method that uses a hyperspectral detector to capture a hyperspectral dataset of diffraction patterns (diffractograms) in a single ptychographic acquisition. ${ }^{2}$ The present article focuses on an improvement on this same method via undulator tapering (see section 2). It also investigates a different sample.

Conceptually the hyperspectral detection capabilities move the longitudinal aspect of the coherence requirements from the experimental domain to the post-processing domain. These relaxed experimental requirements have the potential to bring ptychography from synchrotron facilities to small laboratory setups, which has recently been demonstrated for the first time. ${ }^{3}$ The data described in this article was however acquired at a synchrotron, where more precise control over the radiation spectrum is possible.

In addition to the relaxing of experimental requirements, the extra energy dimension creates an opportunity for single acquisition elemental mapping by edge subtraction, which is what this article focuses on. A caveat that comes with hyperspectral detectors is that because they measure the total energy incident per pixel per sampling interval, they can only function properly in conditions of relatively low flux (compared to the detector temporal and spatial sampling), where photon pile-up events are unlikely. In our case the flux of a polychromatic source should not exceed approximately 5 photons per pixel per second. This requirement naturally increases the necessary exposure time.

Send correspondence to Wiebe Stolp: Wiebe.Stolp@UGent.be 


\section{METHODS}

\subsection{Setup}

The described experiment was carried out at the Diamond Light Source I-13 beamline, at the branch line for $\mathrm{X}$-ray Imaging and Coherence that is specialized in reciprocal space imaging such as ptychography. ${ }^{4}$ Because the energy resolution of the hyperspectral detector is significantly larger than the bandwidth of the undulator harmonics (respectively approximately $140 \mathrm{eV}$ versus $27 \mathrm{eV}$ ), for the purpose of this experiment the undulator was tapered. Tapering refers to the introduction of an angle between the two (usually parallel) sides of the undulator, which broadens the spectral width of the outgoing radiation. By centering an undulator harmonic over an absorption edge and increasing the spectral width using tapering, the spectrum can be optimized for edge subtraction using our hyperspectral detector.

The experimental setup is shown in figure 1 . To provide a suitable probe size, the incoming X-ray beam is focused using a diffractive Fresnel Zone Plate (FZP). The center of the X-ray beam is blocked by a Central Stop (CS) to make better use of the low dynamic range of the detector and to reject the zero order diffraction. This blocking is later corrected for in the reconstruction algorithm by 'floating' the unilluminated pixels. ${ }^{5}$ All higher order diffraction is rejected using an Order Sorting Aperture (OSA).

A difficulty arises: because the FZP focal length is wavelength-dependent, the probe size at the sample stage inherits a strong wavelength dependency as well. Conventional CDI requires a probe size that is inversely proportional to the angular detector pixel pitch. A probe size that satisfies this CDI criterion at a certain central energy, will not do so anymore at an energy that is too high or too low. When a probe is too small, the resulting lack of overlap at the sample stage renders a data set unreconstructable at that energy. Probes that are too wide may in principle be solved in ptychography to some extent in the reconstruction stage via upsampling, albeit at a computational expense. ${ }^{6,7}$

However, at very high or low energies, the probe will be too large at the OSA plane and the OSA will clip it, deteriorating the probe by diffracting from the edges and breaking the lateral coherence. As a consequence, the range of photon energies for which diffractograms can be meaningfully captured in a single acquisition is limited.

With this setup an attempt has been made to perform a K-edge subtraction on a zeolite particle containing Bromine (K-edge $13.474 \mathrm{keV}$ ).

\subsection{SLcam}

The hyperspectral detector used to capture the diffraction patterns is the SLcam. ${ }^{8,9}$ It has a frame rate of $400 \mathrm{~Hz}$ and a functional X-ray sensitive area of $12.7 \mathrm{~mm}^{2}$ distributed over $264 \times 264$ pixels of $48 \mathrm{\mu m}^{2}$ each. Employing a $450 \mathrm{~mm}$ depleted Silicon layer, it functions like a charge coupled device, collecting and measuring electron charge clouds generated by absorbed photons during each sampling interval.

\subsection{Reconstruction}

Ptychographic reconstruction is done using the PtyREX Python package ${ }^{10}$ based on the iterative ePIE algorithm. ${ }^{11}$ Oversized probes are accommodated using upsampling. ${ }^{7}$ A spectral bandwidth is selected from the energy data cubes (diffractograms with an energy dimension) output by the detector, defining a balance between photon statistics and longitudinal coherence. All photon events within this bandwidth are summed together to form one set of diffractograms that is subsequently fed into PtyREX to create a reconstruction.

\section{RESULTS}

Figure 2 shows reconstructions of the Bromine sample. One is done with an energy below the K-edge with a selected spectral bandwidth of $60 \mathrm{eV}$. After repeated attempts at reconstructing we were not able to obtain a higher energy reconstruction that is sufficiently artifact free to perform a meaningful edge subtraction. The reconstruction at the top row is done exactly centered at the K-edge (spectral bandwidth $50 \mathrm{eV}$ ) to see whether an artifact free reconstruction would converge at this limit, to no avail. This might be related to instabilities in the sample during acquisition, creating inconsistencies in the recorded reciprocal space dataset and preventing the reconstruction algorithm to converge. However, the problem is most certainly exacerbated by the probe size limitations described earlier. Future experiments will therefore benefit from achromatic focusing methods. 


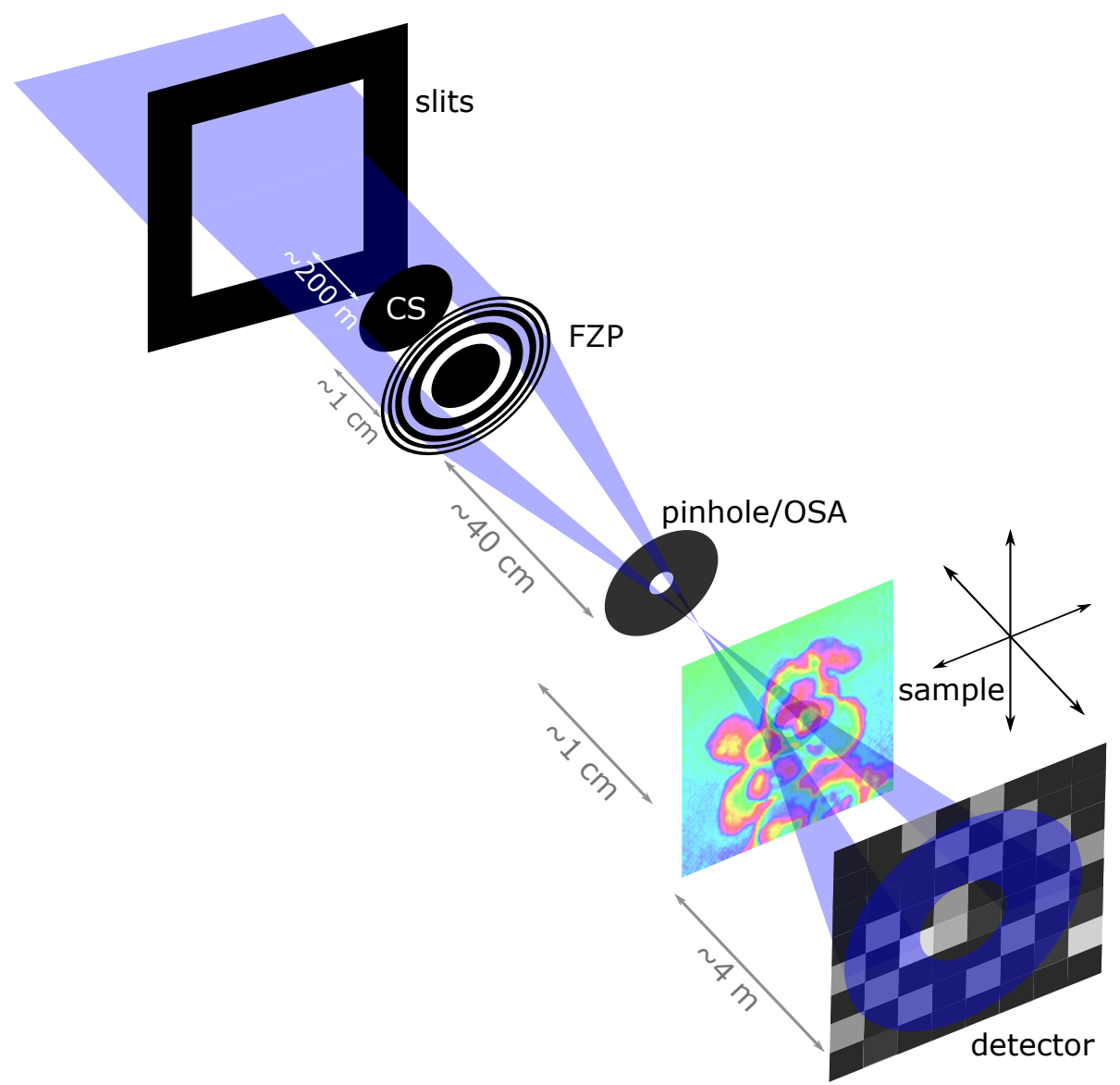

Figure 1. Experimental setup. CS: Central Stop, OSA: Order Sorting Aperture, FZP: Fresnel Zone Plate. 

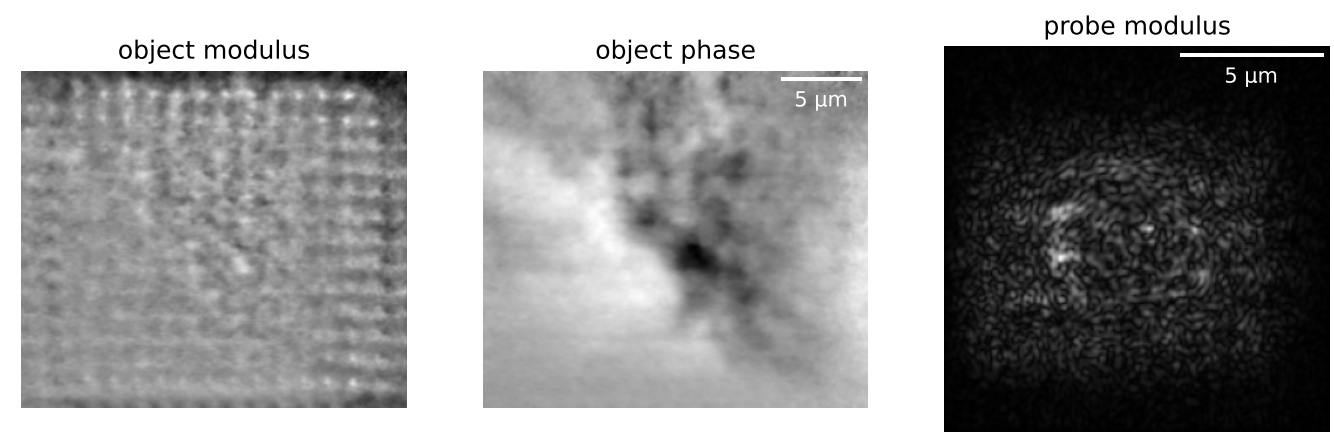

object modulus

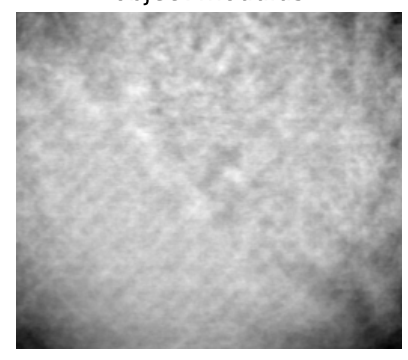

object phase

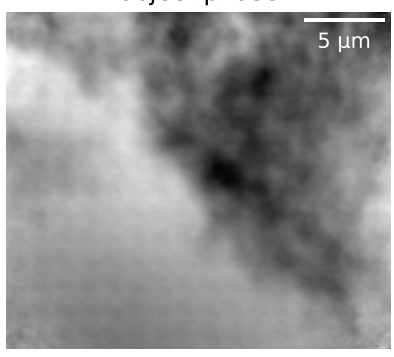

probe modulus

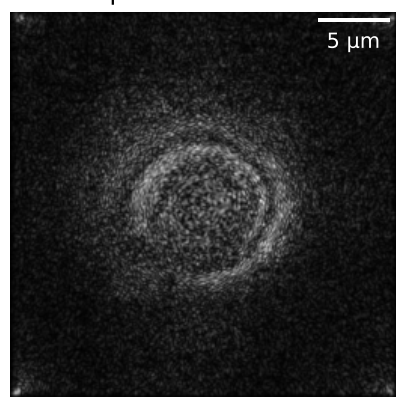

Figure 2. Top row: reconstruction at $13.47 \mathrm{keV}$, exactly at the Br K-edge energy. Bottom row: reconstructions at 13.33 $\mathrm{keV}$, below the Br K-edge energy. The modulus image in the top row is governed by artifacts and any reconstruction at an even higher energy would therefore not be suitable for edge subtraction. 


\section{ACKNOWLEDGMENTS}

Thanks to Dr. Thomas Sheppard from Karlsruhe Institute of Technology for providing the Bromine sample.

\section{REFERENCES}

[1] Maiden, A. M., Morrison, G. R., Kaulich, B., Gianoncelli, A., and Rodenburg, J. M., "Soft X-ray spectromicroscopy using ptychography with randomly phased illumination," Nature Communications 4 (2013).

[2] Batey, D. J., Cipiccia, S., Van Assche, F., Vanheule, S., Vanmechelen, J., Boone, M. N., and Rau, C., "Spectroscopic imaging with single acquisition ptychography and a hyperspectral detector," Scientific Reports $\mathbf{9}(1), 1-7$ (2019).

[3] Batey, D. J., Van Assche, F., Vanheule, S., Boone, M. N., Parnell, A. J., Mykhaylyk, O. O., Rau, C., and Cipiccia, S., "X-ray Ptychography with a Laboratory Source," 1-14 (2021).

[4] Rau, C., Wagner, U., Peach, A., Robinson, I. K., Singh, B., Wilkin, G., and Jones, C., "The diamond beamline I13L for imaging and coherence," AIP Conference Proceedings 1234(2010), 121-125 (2010).

[5] Maiden, A. M., Humphry, M. J., Zhang, F., and Rodenburg, J. M., "Superresolution imaging via ptychography," Journal of the Optical Society of America A 28(4), 604 (2011).

[6] Edo, T. B., Batey, D. J., Maiden, A. M., Rau, C., Wagner, U., Pešić, Z. D., Waigh, T. A., and Rodenburg, J. M., "Sampling in x-ray ptychography," Physical Review A - Atomic, Molecular, and Optical Physics 87(5), 1-8 (2013).

[7] Batey, D. J., Edo, T. B., Rau, C., Wagner, U., Pešić, Z. D., Waigh, T. A., and Rodenburg, J. M., "Reciprocal-space up-sampling from real-space oversampling in x-ray ptychography," Physical Review A - Atomic, Molecular, and Optical Physics 89(4), 1-5 (2014).

[8] Ordavo, I., Ihle, S., Arkadiev, V., Scharf, O., Soltau, H., Bjeoumikhov, A., Bjeoumikhova, S., Buzanich, G., Gubzhokov, R., Günther, A., Hartmann, R., Holl, P., Kimmel, N., Kühbacher, M., Lang, M., Langhoff, N., Liebel, A., Radtke, M., Reinholz, U., Riesemeier, H., Schaller, G., Schopper, F., Strüder, L., Thamm, C., and Wedell, R., "A new pnCCD-based color X-ray camera for fast spatial and energy-resolved measurements," Nuclear Instruments and Methods in Physics Research, Section A: Accelerators, Spectrometers, Detectors and Associated Equipment 654(1), 250-257 (2011).

[9] Bjeoumikhov, A., Buzanich, G., Langhoff, N., Ordavo, I., Radtke, M., Reinholz, U., Riesemeier, H., Scharf, O., Soltau, H., and Wedell, R., "The SLcam: A full-field energy dispersive X-ray camera," in [Journal of Instrumentation], 7, 1-5 (2012).

[10] Batey, D., "Ptychographic imaging of mixed states," (September) (2014).

[11] Maiden, A. M. and Rodenburg, J. M., "An improved ptychographical phase retrieval algorithm for diffractive imaging," Ultramicroscopy 109(10), 1256-1262 (2009). 